12

\title{
Исследование квадрупольного фильтра масс с квадрупольным возбуждением методом уравнения огибающей
}

\author{
(C) М.Ю. Судаков, Е.В. Мамонтов
}

Рязанский государственный радиотехнический университет, 390035 Рязань, Россия

e-mail: m.soudakov@yandex.ru

(Поступило в Редакцию 29 февраля 2016 г.)

Развит метод уравнения огибающей для описания стабильности движения ионов в квадрупольном фильтре масс при наличии периодических возмущений питающего напряжения. Вблизи вершины первой общей зоны стабильности получены динамические уравнения, описывающие изменения огибающей колебаний ионов, и приведены к форме уравнений Матье. Описано расщепление диаграммы стабильности движения ионов на островки стабильности за счет возбуждения. Результаты приближенной теории подтверждаются точными расчетами диаграммы стабильности при рациональных значениях относительной частоты возбуждения. Определены рамки применимости развитой теории, которые ограничены резонансами первого порядка.

\section{Введение}

Со времени открытия В. Паулем [1] масс-селективной сортировки ионов в переменных электрических полях ионные ловушки и квадрупольные фильтры масс получили широкое развитие в качестве сравнительно недорогих и компактных масс спектрометров. В настоящее время квадруполи рутинно применяются в технике физического эксперимента для анализа состава остаточных газов, в составе тандемных инструментов для предварительной сортировки ионов по массам, а также в качестве проводников ионных пучков и столкновительных ячеек для расщепления родительских ионов на фрагменты.

Фильтрация ионов в квадруполях основана на свойствах уравнения Матье, которым описывается движение ионов в квадрупольном поле при гармоническом питающем напряжении [2]. Поэтому технология квадруполей требует изготовления и сборки электродов гиперболического профиля с микронной точностью, а также разработки стабильных и управляемых источников высокочастотного (ВЧ) и высоковольтного гармонического питания. Несмотря на хорошо развитую технологию квадруполей, ведется поиск и исследование новых нестандартных методов их работы. Так, в лаборатории проф. Д. Дугласа из Университета Британской Колумбии исследовались квадруполи с искаженной формой электродов $[3,4]$ и при наличии периодических возмущений питающего напряжения [5,6]. Несмотря на общепринятое представление о том, что эффективная фильтрация ионов возможна только при использовании высококачественных квадрупольных полей [7-10], было обнаружено, что квадруполи с сильно искаженным полем могут использоваться для фильтрации ионов с неменьшим успехом [3]. Более того, использование дополнительных периодических возмущений ВЧ-питания позволяет преодолеть деструктивное влияние искажений поля и получить в квадруполях с сильно искаженной формой электродов разрешающую способность и пропускание ионов, сравнимые с коммерческими образцами [6].
В настоящей работе развивается теория уравнений огибающей [11] для описания движения и устойчивости ионов в квадрупольном ВЧ-поле при наличии периодических возмущений питающего напряжения. Будет показано, что исходная диаграмма стабильности Матье разбивается на полосы стабильности, а совместная диаграмма стабильности движения ионов по двум пространственным направлениям соответственно разбивается на островки стабильности, которые можно использовать для фильтрации ионов. Периодические возмущения питающего напряжения приводят к параметрическому резонансу на частотах секулярных колебаний ионов. Уравнение огибающих позволяет описать это явление и соответствующее расщепление диаграммы стабильности с помощью уравнений Матье, в которых параметры стабильности пропорциональны амплитуде периодического возмущения питания. Результаты приближенной теории проверяются точным расчетом диаграммы стабильности в случаях, когда относительная частота возбуждения представляется рациональным числом.

\section{1. Уравнения движения ионов в фильтре масс при наличии периодических возмущений питающего напряжения}

Квадрупольный фильтр масс образован четырьмя стержнями с гиперболическим профилем, расположенными симметрично относительно общей оси $(Z)$. При приложении питания (положительная фаза к паре противоположных стержней, расположенных вдоль направления $X$, а отрицательная - к паре стержней вдоль $Y$ ) в пространстве между стержнями образуется переменное квадрупольное поле

$$
\Phi(x, y)=V(t) \cdot \frac{x^{2}-y^{2}}{r_{0}^{2}} .
$$


Здесь $r_{0}-$ радиус окружности, вписанной между электродами фильтра („радиус поля“). При обычной работе квадруполя периодическая функция питания $V(t)$ равна

$$
V(t)=U+V \cos \left\lfloor\Omega\left(t-t_{0}\right)\right\rfloor .
$$

Здесь $U$ и $V$ - постоянная и амплитуда переменной составляющих периодического питающего напряжение на электродах фильтра, $\Omega$ - частота основного питания, a $t_{0}-$ начальная фаза. Движение ионов массой $m \mathrm{c}$ зарядом $e$ в таком поле описывается парой уравнений Матье, которые обычно записывают с использованием безразмерных параметров

$$
\begin{aligned}
& \frac{d^{2} x}{d \xi^{2}}=(a+2 q \cos 2 \xi) \cdot x=0, \\
& \frac{d^{2} y}{d \xi^{2}}=(a+2 q \cos 2 \xi) \cdot y=0,
\end{aligned}
$$

где

$$
\xi=\frac{\Omega}{2}\left(t-t_{0}\right), \quad a=\frac{8 e U}{m \Omega^{2} r_{0}^{2}} \quad \text { и } \quad q=\frac{4 e V}{m \Omega^{2} r_{0}^{2}} .
$$

Свойства уравнения Матье хорошо изучены [12]. Известно, что на плоскости параметров $(a, q)$ имеются области стабильности, разделенные областями нестабильности (хорошо известная диаграмма Айнса [13]), в которых решения уравнения (3a) экспоненциально нарастают. Параметры в уравнении движения вдоль $Y$ имеют противоположный знак, поэтому соответствующая диаграмма стабильности является зеркальным отражением диаграммы рис. 1 относительно осей $a$ и $q$. Для удержания ионов в поле квадруполя практическое значение имеют области совместной стабильности движения по обоим направлениям. Первая из таких областей показана на рис. 2. В точке $a_{1}=0.236993, q_{1}=0.705996$ сходятся две границы областей стабильности движения слева по $Y$ и справа по $X$. Из уравнений (4) очевидно, что для ионов разной массы параметры стабильности лежат на одной „рабочей“ прямой $a=2 \lambda q$, где $\lambda=U / V$, причем чем тяжелее ион, тем меньше соответствующие параметры. Принцип фильтрации ионов по массе состоит в использовании таких величин питающих напряжений, чтобы рабочая прямая проходила вблизи вершины зоны стабильности (рис. 2). Тогда при пропускании пучка ионов вдоль оси фильтра на выход пройдут только те ионы, массы которых попадают в узкий диапазон между границами стабильности, остальные осядут на электродах. При этом более тяжелые ионы испытывают нестабильность движения вдоль $Y$ и попадают на $Y$ стержни, а более легкие - на $X$ стержни.

При наличии периодических возмущений питания функция $V(t)$ имеет дополнительные слагаемые. В простейшем случае квадрупольного возбуждения на частоте $\omega$ с амплитудой $V_{\mathrm{ex}}$ она содержит слагаемое

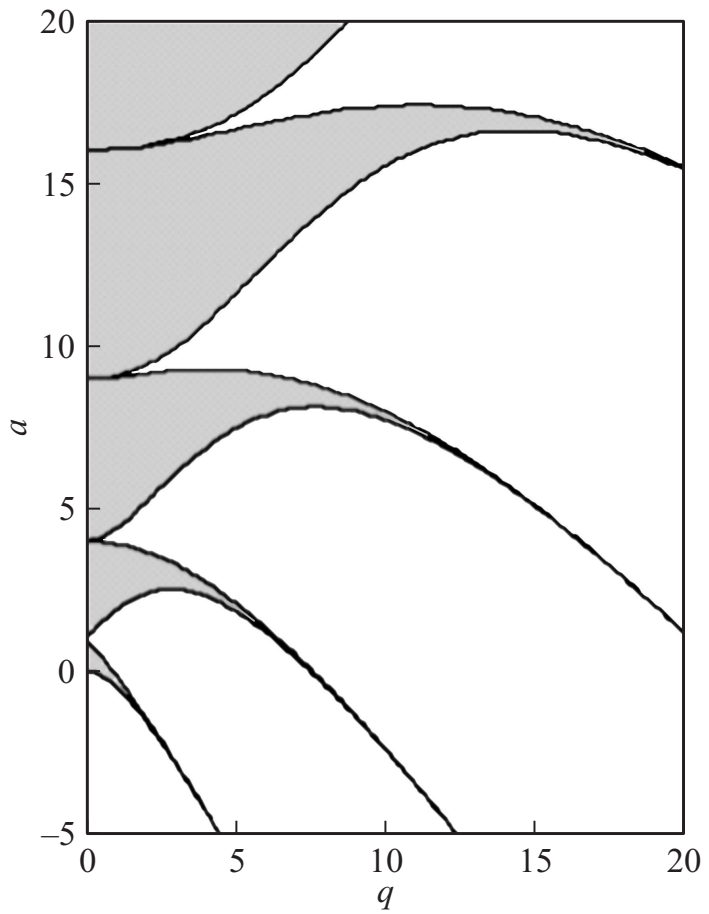

Рис. 1. Диаграмма стабильности уравнения Матье. Стабильные области помечены серым цветом, границы областей жирными линиями. При отрицательных значениях параметра $q$ диаграмма имеет симметричный вид.

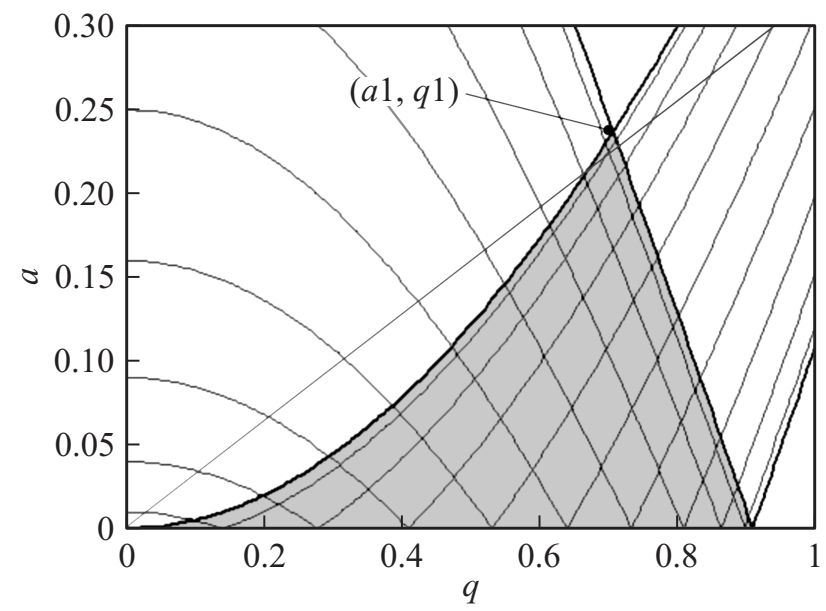

Рис. 2. Первая зона стабильности совместной диаграммы квадрупольного фильтра масс. Зоны стабильных движений показаны серым цветом. Тонкими черными линиями показаны изолинии параметра $\beta$ с шагом 0.1. Тонкая линия - рабочая прямая.

$V_{\text {ex }} \cos \left\lfloor\omega\left(t-t_{0}\right)+\alpha\right\rfloor$. В этом случае уравнения движения усложняются и в безразмерных единицах имеют вид

$$
\frac{d^{2} x}{d \xi^{2}}+\left[a+2 q \cos 2 \xi+2 q_{\mathrm{ex}} \cos (2 v \xi+\alpha)\right] \cdot x=0,
$$

где

$$
v=\frac{\omega}{\Omega} \quad \text { и } \quad q_{\mathrm{ex}}=\frac{4 e V_{\mathrm{ex}}}{m \Omega^{2} r_{0}^{2}} .
$$




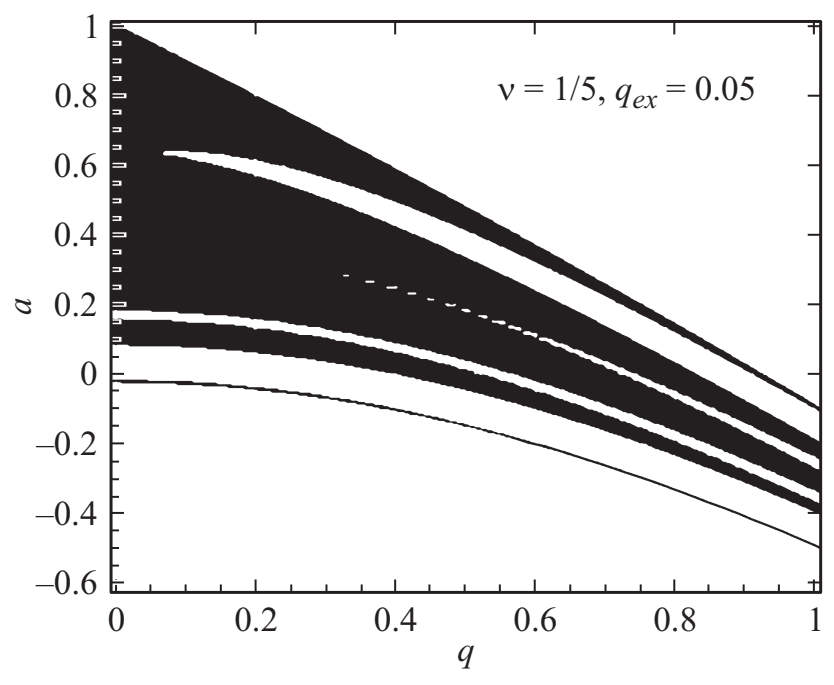

Рис. 3. Расщепление первой зоны стабильности движения вдоль направления $X$ под воздействием квадрупольного возбуждения на частоте $1 / 5$ от основной частоты ВЧ с амплитудой $q_{\mathrm{ex}}=0.05$. Стабильные области показаны черным цветом.

Ранее было показано [14], что квадрупольное возбуждение изменяет свойства стабильности движения ионов и под его влиянием диаграмма стабильности Матье разбивается на полосы нестабильности (рис. 3). Число полос конечно и зависит от частоты возбуждения $\omega$, а ширина полос нестабильности тем больше, чем выше амплитуда возбуждения $V_{\text {ex }}$.

В этой работе не преследуется цель изучить все возможные случаи периодических возмущений ВЧ-питания, такие как модуляция переменной составляющей и всего питающего напряжения, фазовая и частотная модуляции и случай двух квадрупольных возбуждений на разных частотах. Исследование таких возмущений, несомненно, важно для практики, но уместно для другой публикации. Влияние одиночного возбуждения, исследуемое в настоящей работе, может служить основой для этого. Мы также не можем предложить исчерпывающего исследования расщепления диаграммы стабильности сигналами всевозможных частот и амплитуд. Вместо этого мы намерены изучать расщепление диаграммы вблизи вершины первой зоны стабильности, что наиболее важно для практики фильтров масс. Ниже будет показано, что большого прогресса в этой задаче можно достигнуть с использованием теории возмущений и метода уравнений огибающей колебаний ионов.

\section{2. Теория возмущений для уравнения Матье}

Уравнение (5) можно представить в виде

$$
\frac{d^{2} x}{d \xi^{2}}=[a+2 q \cos 2 \xi] \cdot x=f(\xi) \cdot x
$$

где в правой части уравнения выделено возмущение в виде функции

$$
f(\xi)=-2 q_{\mathrm{ex}} \cdot \cos (2 \nu \xi+\alpha)
$$

Расчеты показывают, что даже малые величины возбуждений $q_{\mathrm{ex}}$ приводят к возникновению значительных полос нестабильности на исходной диаграмме, особенно в тех случаях, когда относительная частота возбуждения $v$ мала или близка к единице (т.е. фактическая частота близка к частоте основного ВЧ). Поэтому оправданным является применение теории возмущений, в которой слагаемое в правой части уравнения (7) считается малым по сравнению с остальными. Использование теории возмущений для вычисления решений на больших интервалах времени приведет к большой погрешности, так как возмущенное решение может далеко отклониться от исходного. Здесь целесообразно вычислить возмущение на одном периоде ВЧ-поля и использовать матричное преобразование координат для вычисления решения спустя много циклов ВЧ. В этом отношении данный подход аналогичен методам, используемым в технике циклических ускорителей, где для анализа динамики частиц вычисляют матрицу преобразования координат частиц с учетом аберраций на одном проходе через систему и далее используют это преобразование для того, чтобы определить координаты частиц спустя много проходов. Действительно, движение ионов в ВЧ-полях во многом схоже с движением частиц в ускорителях, так как и там, и там частицы периодически испытывают воздействие одинакового по форме поля.

В отсутствие возмущений уравнения движения ионов являются уравнениями Матье, т.е. линейными дифференциальными уравнениями с коэффициентами, периодически зависящими от времени. Теоретический анализ таких уравнений можно проводить различными методами, основываясь на теореме Флоке-Ляпунова [15], или путем представления решения в виде ряда Фурье и прямого вычисления коэффициентов ряда, как изложено в [12], методами, развитыми в работах $[16,17]$, однако здесь для нас наиболее удобен матричный метод, развитый в [18]. Решение уравнения (3a), как и решение любого линейного дифференциального уравнения второго порядка, можно представить в виде линейной комбинации двух независимых частных решений $u_{1}(\xi)$ и $u_{2}(\xi)$. Последние удобно определить с помощью начальных условий вида

$$
u_{1}(0)=1, \quad u_{1}^{\prime}(0)=0 \quad \text { и } \quad u_{2}(0)=0, \quad u_{2}^{\prime}(0)=1 .
$$

Здесь штрих означает производную по безразмерной переменной $\xi$. Общее решение с начальной координатой $x_{0}$ и скоростью $v_{0}$ тогда равно

$$
x(\xi)=x_{0} \cdot u_{1}(\xi)+v_{0} \cdot u_{2}(\xi) .
$$


Из уравнения (10) можно получить координату $x_{1}$ и скорость $v_{1}$ частицы спустя один период ВЧ в виде

$$
\begin{aligned}
& x_{1}=m_{11} x_{0}+m_{12} v_{0}, \\
& v_{1}=m_{21} x_{0}+m_{22} v_{0} .
\end{aligned}
$$

Здесь введены обозначения для коэффициентов так называемой матрицы монодромии:

$$
\mathbf{M}=\left[\begin{array}{ll}
u_{1}(\pi) & u_{2}(\pi) \\
u_{1}^{\prime}(\pi) & u_{2}^{\prime}(\pi)
\end{array}\right]=\left[\begin{array}{ll}
m_{11} & m_{12} \\
m_{21} & m_{22}
\end{array}\right]
$$

которые вычисляются через значения частных решений в конце периода.

Из-за периодичности коэффициентов уравнения (3а) оно имеет те же самые частные решения $u_{1}(\xi)$ и $u_{2}(\xi)$, если переменная $\xi$ отсчитывается от начала следующего периода. Поэтому значения координат частицы в конце каждого периода можно определить по координатам в начале периода с помощью соотношений, подобных (11) с теми же самыми коэффициентами. Таким образом, пара решений $u_{1}(\xi)$ и $u_{2}(\xi)$, вычисленных на протяжении всего одного периода, позволяет полностью характеризовать решение уравнения (3a) в любой момент времени. В частности, стабильность решения зависит от величины следа матрицы монодромии $m_{11}+m_{22}$. Решение является стабильным, если величина следа по модулю не превышает двойки. Для стабильных решений фундаментальное значение имеет „секулярная“ частота $\omega_{s}=\beta \Omega / 2$, которая связана с безразмерным параметром $\beta$. Последний вычисляется через след матрицы монодромии из уравнения

$$
\cos (\pi \beta)=\frac{1}{2}\left(m_{11}+m_{22}\right) .
$$

Нашей дальнейшей задачей является вычисление матричного преобразования координат, подобного (11), с учетом возмущения. Для возмущений такого вида, как в уравнении (7) в работе [19], в первом порядке получено выражение, связывающее координаты в конце с координатами в начале $n$-го периода в виде

$$
\left(\begin{array}{l}
x_{n+1} \\
v_{n+1}
\end{array}\right)=\mathbf{M} \times\left\{1-\int_{0}^{\pi} f \cdot\left[\begin{array}{cc}
u_{1} u_{2} & u_{2}^{2} \\
-u_{1}^{2} & -u_{1} u_{2}
\end{array}\right] d \tau\right\}\left(\begin{array}{l}
x_{n} \\
v_{n}
\end{array}\right) .
$$

Здесь решения $u_{1}(\tau)$ и $u_{2}(\tau)$ являются функциями переменной интегрирования $\tau$, которая отсчитывается от начала $n$-го периода: $\xi=n \pi+\tau$, а подынтегральное выражение для функции возмущения $f(\xi)$, согласно уравнению (8), можно представить в виде

$$
\begin{aligned}
f= & -2 q_{\mathrm{ex}} \cdot \cos \lfloor 2 v(n \pi+\tau)+\alpha\rfloor \\
= & -2 q_{\mathrm{ex}} \cdot[\cos (2 v n \pi+\alpha) \cos (2 \nu \tau) \\
& -\sin (2 \nu n \pi+\alpha) \sin (2 \nu \tau)] .
\end{aligned}
$$

Тогда результирующую матрицу преобразования в уравнении (14) можно преобразовать к виду

$$
\mathbf{M}_{n}=\mathbf{M}+\mathbf{C} \cdot \cos (2 \nu n \pi-\alpha)-\mathbf{S} \cdot \sin (2 \nu n \pi-\alpha),
$$

где $\mathbf{C}$ и $\mathbf{S}$ являются постоянными матрицами

$$
\begin{aligned}
& \mathbf{C}=2 q_{\mathrm{ex}} \cdot \mathbf{M} \times\left[\begin{array}{cc}
c_{12} & c_{22} \\
-c_{11} & -c_{12}
\end{array}\right], \\
& \mathbf{S}=2 q_{\mathrm{ex}} \cdot \mathbf{M} \times\left[\begin{array}{cc}
s_{12} & s_{22} \\
-s_{11} & -s_{12}
\end{array}\right] .
\end{aligned}
$$

Здесь

$$
\begin{gathered}
c_{i, j}=\int_{0}^{\pi} \cos 2 v \tau \cdot u_{i}(\tau) u_{j}(\tau) d \tau, \\
s_{i, j}=\int_{0}^{\pi} \sin 2 v \tau \cdot u_{i}(\tau) u_{j}(\tau) d \tau, \quad i, j=1,2 .
\end{gathered}
$$

Уравнение (16) показывает, что в данном случае отображение (14) является рекуррентным матричным преобразованием с коэффициентами, периодически изменяющимися с номером периода $n$. Анализ свойств таких отображений выходит за рамки данной публикации, хотя можно предположить, что свойства его решений во многом подобны свойствам решений дифференциальных уравнений с периодическими коэффициентами. В частности, будут возникать параметрические нестабильности при определенных соотношениях между частотой возмущения $v$ и секулярной частотой невозмущенного движения, а именно с безразмерным параметром $\beta$. В следующем разделе будет установлена эта связь для случаев, когда значения параметра $\beta$ близки к границам стабильности.

В заключение этого раздела заметим, что для движения вдоль координаты $Y$ уравнения движения имеют аналогичный вид, однако параметры как питающего напряжения, так и возмущения, отличаются противоположным знаком. Для движения вдоль $Y$ справедливы аналогичные рассуждения с тем отличием, что частные решения $u_{1}(\xi)$ и $u_{2}(\xi)$ будут, разумеется, другими. В дальнейшем, где это необходимо, функции и величины будут обозначаться индексами $x$ и $y$.

\section{3. Уравнение огибающей для описания траекторий ионов при движении вблизи границ стабильности}

Движение ионов при параметрах, лежащих вблизи границ зоны стабильности, обладает особенностями, которые можно использовать для упрощенного описания движения. В частности, вблизи границы $\beta=0$ (что характерно для движения вдоль $Y$ ) значения координат иона, взятые через период, очень мало отличаются 
друг от друга, поэтому можно описывать последовательность координат некоторой плавной функцией $y_{n}=Y(n)$. Очень похожая ситуация имеет место при движении иона вблизи другой границы стабильности $\beta=1$ (движение вдоль $X$ ). Здесь движение иона носит характер биений с частотой, равной половине частоты питающего ВЧ. Координаты иона, взятые через период (стробоскопические), противоположны по знаку и почти равны по абсолютной величине. В этом случае тоже можно ввести в рассмотрение некоторую плавную функцию $X(n)$ и использовать приближение $x_{n}=(-1)^{n} X(n)$. На этом основано приближение уравнений огибающих, развитое в работах $[11,20]$. Исследование движений ионов вблизи границ стабильности имеет особое значение, так как работа фильтра масс осуществляется вблизи вершины первой диаграммы стабильности, где сходятся две границы стабильности $\beta_{x}=1$ и $\beta_{y}=0$. Таким образом, использование особых свойств движения ионов вблизи границ стабильности позволяет продвинутся вперед в теоретическом исследовании влияний квадрупольных возбуждений на работу фильтра масс.

В работе [11] показано, что огибающая биений, которые испытывает ион при движении вдоль направления $X$ вблизи границы стабильности $\beta_{x}=1$, подчиняется уравнению

$$
\frac{d^{2} X(n)}{d n^{2}}+2[1+s] \cdot X(n)=0
$$

где $s=0.5 \cdot\left(m_{11}+m_{12}\right)$ - половина следа матрицы монодромии. В случае, когда имеется возмущение движения, матрица преобразования, как и ее след, зависит от номера периода $n$. Вычислим зависимость параметра $s(n)$ для случая квадрупольного возбуждения, используя уравнение (16). При этом в первом слагаемом используем фактическое значение матрицы монодромии невозмущенного движения М, а при вычислении второго и третьего слагаемых в уравнении (16) будем считать, что матрица монодромии не отличается от своего значения на границе, которое равно

$$
\mathbf{M}_{x}=\left[\begin{array}{cc}
-1 & \Pi_{x} \\
0 & -1
\end{array}\right],
$$

где $\Pi_{x}-$ некоторое число $\left(\Pi_{x}=3.7502787\right.$ в вершине первой зоны). Такое приближение обосновано тем, что в методе огибающей отклонение $\Delta a=a-a_{1}$ и $\Delta q=q-q_{1}$ параметров $a$ и $q$ от их значений на границе стабильности тоже является малым параметром такого

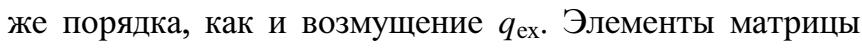
монодромии отличаются от своих значений на границе стабильности пропорционально этим отклонениям. Поэтому при вычислении частей, связанных с возмущением в уравнении (16), отклонения фактической матрицы монодромии М от выражения (20) попадают во второй порядок теории возмущений. С учетом вышесказанного, вычисление на основе уравнения (16) дает

$$
\begin{aligned}
s(n)= & s_{0, x}-q_{\mathrm{ex}} \Pi_{x} \cdot\left\lfloor c_{11, x} \cos (2 v n \pi-\alpha)\right. \\
& \left.-s_{11, x} \sin (2 v n \pi-\alpha)\right\rfloor
\end{aligned}
$$

где $s_{0, x}=0.5 \cdot\left(m_{11}+m_{22}\right)$ - параметр для невозмущенного движения.

Пользуясь тригонометрическими тождествами, выражение (21) можно представить в виде

$$
s(n)=s_{0, x}-q_{\mathrm{ex}} \cdot F_{x} \cdot \cos \left(2 v n \pi-\alpha^{*}\right),
$$

где

$$
F_{x}=\Pi_{x} \sqrt{\left(c_{11, x}\right)^{2}+\left(s_{11}, x\right)^{2}} \text { и } \alpha^{*}=\alpha+\operatorname{arctg} \frac{s_{11, x}}{c_{11, x}} .
$$

Подставляя полученное выражение в уравнение огибающей (19), получим

$$
\frac{d^{2} X}{d n^{2}}+\left[2\left(1+s_{0, x}\right)-2 q_{\mathrm{ex}} F_{x} \cdot\left(2 v n \pi-\alpha_{x}\right)\right] \cdot X(n)=0 .
$$

Заметим, что в уравнении (24) периодическая функция, стоящая в скобках, не меняет своего значения при добавлении к частоте $v$ любого целого числа. Поэтому в тех случаях, когда относительная частота возбуждения $v$ близка к целому числу $k$, следует использовать „приведенное“ значение частоты

$$
\tilde{v}=v-k
$$

Введем независимую переменную $2 \xi=2 \tilde{v} n \pi-\alpha^{*}$. Тогда окончательно уравнение огибающей запишется в виде

$$
\frac{d^{2} X}{d \zeta^{2}}+\left[A_{x}+2 Q_{x} \cdot \cos 2 \zeta\right] \cdot X=0
$$

где

$$
A_{x}=\frac{2\left(1+s_{0, x}\right)}{\pi^{2} \tilde{v}^{2}} \quad \text { и } \quad Q_{x}=-\frac{q_{\mathrm{ex}} F_{x}}{\pi^{2} \tilde{v}^{2}} .
$$

Полученное уравнение имеет вид уравнения Матье и с его помощью можно описать разбиение исходной диаграммы на полосы нестабильности со стороны границы $\beta_{x}=1$.

Прежде чем переходить к анализу результатов развитой теории, необходимо исследовать влияние возмущения на движение ионов при параметрах, близких к границе $\beta=0$. Такие параметры в фильтре масс характерны для движения вдоль $Y$. Как уже отмечалось, в этом случае стробоскопические координаты $y_{n}$, взятые через период ВЧ, мало отличаются друг от друга по величине и можно ввести функцию огибающей колебаний $y_{n} \approx Y(n)$. В уже цитированной работе [11] показано, что огибающая в этом случае подчиняется уравнению

$$
\frac{d^{2} Y(n)}{d n^{2}}+2[1-s] \cdot Y(n)=0 .
$$


Заметим, что матрица монодромии на границе стабильности $\beta_{y}=0$ имеет вид, несколько отличающийся от (20):

$$
\mathbf{M}_{y}=\left[\begin{array}{cc}
1 & \Pi_{y} \\
0 & 1
\end{array}\right],
$$

где $\Pi_{y}-$ некоторое число, зависящее от конкретного положения на границе $\left(\Pi_{y}=1.7222927\right.$ в вершине первой зоны). Теория возмущений для движения вдоль $Y$ формально выглядит аналогично, за исключением противоположного знака параметра возбуждения $q_{\mathrm{ex}}$, поэтому, повторяя рассуждения, приведшие к уравнению (26), получим для движения вдоль $Y$ следующее уравнение огибающей:

$$
\frac{d^{2} Y}{d \xi^{2}}+\left[A_{y}+2 Q_{y} \cdot \cos 2 \xi\right] \cdot Y=0,
$$

где

$$
A_{y}=\frac{2\left(1-s_{0, y}\right)}{\pi^{2} \tilde{v}^{2}} \quad \text { и } \quad Q_{y}=\frac{q_{\mathrm{ex}} F_{y}}{\pi^{2} \tilde{v}^{2}} .
$$

Здесь $s_{0}=0.5 \cdot\left(m_{11}+m_{22}\right)$ - параметр невозмущенного движения для $Y$, а $F_{y}$ вычисляется по формулам, аналогичным (23), но с использованием фундаментальных решений уравнения (3b).

\section{4. Анализ результатов}

В этом разделе будут исследованы основные следствия уравнений (26) и (29). При этом результаты разработанной приближенной теории сравниваются с точным расчетом, который доступен для уравнения (5) в случаях, когда частота возмущения равна несократимой дроби: $v=K / P$, где $K$ и $P$ - целые числа. В этом случае коэффициенты в уравнениях движения обладают общим периодом $P \cdot \pi$, и для анализа устойчивости решений можно использовать точный метод, впервые предложенный в работе [14]. Он основан на вычислении следа матрицы монодромии на протяжении полного периода $P \cdot \pi$. В приводимых ниже расчетах, построение диаграмм стабильности на плоскости параметров $(a, q)$ производилось путем вычисления следа матрицы монодромии для движений вдоль $X$ и вдоль $Y$ для каждой точки (пиксела) исследуемой области диаграммы. В случае, если оба движения стабильны, то пиксел помечается темным цветом. Вычисление фундаментальных решений уравнения для вычисления матрицы монодромии производилось методом Рунге-Кутты 4-го порядка с постоянным шагом [21]. Точность вычислений контролировалась наблюдением изменений, происходящих в диаграмме при измельчении шага интегрирования. Практика показала, что в исследуемой области параметров и возбуждений шаг $\pi / 32$ достаточен для получения требуемой точности (дальнейшее уменьшение шага интегрирования не изменяет вида диаграммы в пределах изображения).

\section{1. Общая характеристика разбиения диаграммы стабильности}

Первым важным выводом является независимость стабильности частиц от начальной фазы квадрупольного возбуждения. Действительно, она оказалась включенной в определение независимой переменной $\xi$ и никак не сказывается на стабильности решений. Этот вывод подтверждается прямыми расчетами диаграмм стабильности для рациональных значений частоты. При изменении начальной фазы возбуждения вид диаграммы не меняется, хотя форма питающего напряжения очевидно различна. Таким образом, при реализации квадрупольных возбуждений на практике не требуется обеспечивать фазовый синхронизм между возбуждением и основным питанием.

Теоретический анализ разбиения диаграммы стабильности на полосы нестабильности вблизи границы $\beta_{x}=1$ можно провести с помощью уравнения (26). Оно является уравнением Матье и для него справедлива хорошо известная диаграмма стабильности Айнса (рис. 4). При фиксированной частоте возбуждения $v$ параметр $Q_{x}$ является постоянным, чему соответствует вертикальная линия на диаграмме. Из пересечений этой линии с границами стабильности можно найти соответствующие значения параметра $A_{x}: A^{0}, A^{1}, A^{2}, \ldots$ Используя определение параметра $A_{x}$ в уравнении (26), получим, что граница зоны стабильности $A^{k}(k=0,1,2, \ldots)$ описывается уравнением

$$
\frac{2\left(1+s_{0}\right)}{\pi^{2} \tilde{v}^{2}}=A^{k}
$$

Для начала будем считать, что величина $A^{k}>0$. Из определения параметра $\beta$ (уравнение (13)) следует $\cos (\pi \beta)=s_{0}$. Так как в рассматриваемом случае параметр $\beta$ близок к единице, удобно преобразовать это выражение к эквивалентному: $\cos \lfloor\pi(1-\beta)\rfloor=-s_{0}$

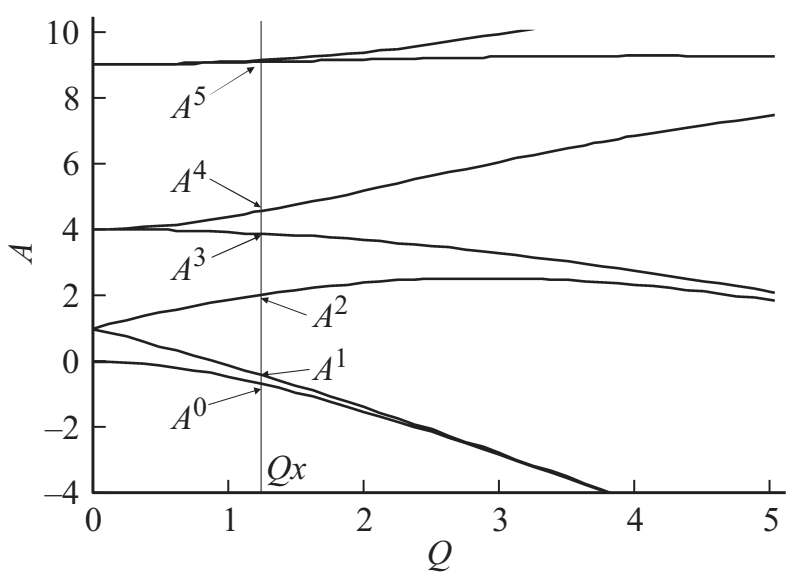

Pис. 4. Диаграмма, иллюстрирующая расчет расщепления зоны на нестабильные полосы. Вертикальная линия проведена при постоянном значении параметра возбуждения $Q_{x}$. Стрелками помечены пересечения этой линии с границами стабильности. 
и воспользоваться тригонометрическим тождеством: $1-\cos (x)=2 \sin ^{2}(x / 2)$. Получим из (30) следующее уравнение

$$
\sin \left[\frac{\pi(1-\beta)}{2}\right]=\frac{\pi \tilde{v}}{2} \sqrt{A^{k}} \quad \text { при } \quad \beta \approx 1 .
$$

Согласно этому уравнению, границы полос нестабильности, на которые разбивается исходная диаграмма стабильности, проходят по изолиниям параметра $\beta$. Это согласуется с расчетами, приведенными, в частности, на рис. 3.

При отрицательных значениях параметра $A^{k}$ (например, $\left.A^{0}<0\right)$ величина $1+s_{0}$ оказывается меньше нуля. Это соответствует нестабильной области на исходной диаграмме: $s_{0}<-1$. В этом случае вместо параметра $\beta$ необходимо использовать инкремент нарастания колебаний $\mu$, который определяется из уравнения $\operatorname{ch}(\mu)=\left|s_{0}\right|$. Для параметра $\mu$ при отрицательных значениях $A^{k}$ можно получить уравнение, подобное (31). Заметим, что граница $A^{0}$ всегда соответствует нестабильной области невозмущенного движения и проходит вдоль изолинии параметра $\mu$. При больших значениях параметра возбуждения $Q_{x}$ не только $A^{0}$, но и $A^{1}$ оказываются отрицательными. Это означает, что на исходной диаграмме новая полоса стабильности лежит целиком в области, которая ранее соответствовала нестабильным движениям. Появление в результате квадрупольного возбуждения полос стабильности в изначально нестабильной области движения является типичной чертой.

На основе уравнения (29) можно получить уравнения для границ полос нестабильности со стороны границы $\beta=0$. После тригонометрических преобразований оно имеет вид

$$
\sin \left[\frac{\pi \beta}{2}\right]=\frac{\pi \tilde{v}}{2} \sqrt{A^{k}} \quad \text { при } \quad \beta \approx 0 .
$$

Здесь результаты аналогичны предыдущим - границы полос идут вдоль изолиний параметра $\beta$, или параметра $\mu$ в нестабильной области.

Из определения параметров $Q_{x}$ и $Q_{y}$ следует, что даже возбуждение очень малой амплитуды может вызвать значительное расщепление диаграммы стабильности, если приведенная частота $\tilde{v}$ близка к нулю. Исследуем случай слабого расщепления, т.е. при малых значениях параметров $Q$. Как видно из рис. 4 , зоны нестабильности в этом случае очень узкие и границы стабильности выходят из одной точки на вертикальной оси, так что справедливо $A^{2 k} \approx A^{2 k-1} \approx k^{2}$. В этом случае из (31) получим уравнение центра полос нестабильности в виде

$$
\sin \left[\frac{\pi(1-\beta)}{2}\right]=\frac{\pi \tilde{v}}{2} k, \quad k=1,2, \ldots
$$

Учитывая, что данное уравнение получено для случая $\beta \approx 1$, его можно еще упростить:

$$
1-\beta=\tilde{v} \cdot k, \quad k=1,2, \ldots
$$

Это уравнение устанавливает условие параметрического резонанса колебаний иона с дополнительным квадру- польным возбуждением. Оно получено вблизи границы $\beta \approx 1$, и его область применимости ограничена условием $\beta<0.5$. Следовательно, количество резонансов ограничено неравенством $k<0.5 / \tilde{v}$.

Аналогично получим условие резонансов со стороны границы $\beta \approx 0$ :

$$
\beta=\tilde{v} \cdot k, \quad k=1,2, \ldots
$$

Здесь также количество резонансов ограничено величиной $k<0.5 / \tilde{v}$. Таким образом, общее число полос нестабильности не превышает целой части от $1 / \tilde{v}$. Этот вывод согласуется с результатами прямого расчета расщепления диаграмм (см., например, рис. 3). Хотя экстраполяция теории возмущений первого порядка на случай параметрического резонанса высоких порядков, вообще говоря, некорректна, развитая теория дает правильное предсказание для общего числа резонансов.

Учитывая, что собственная (секулярная) частота колебаний иона равна $\omega_{s}=\beta \cdot \Omega / 2$, а размерная частота возбуждения $\omega_{\mathrm{ex}}=v \cdot \Omega$, можно придать уравнению (35) обычный для параметрического резонанса вид: $\omega_{s}=2 \omega_{\mathrm{ex}} \cdot k$. Таким образом, прослеживается прямая аналогия квадрупольного возбуждения с обычным параметрическим резонансом, где в качестве частоты осциллятора выступает секулярная частота колебаний иона.

Приводимые здесь условия резонанса, так же как и свойства разбиения диаграммы стабильности на полосы, были известны ранее на основе расчета диаграмм для рациональных значений частоты возбуждения $v=K / P$. Новым результатом, следующим из развитой здесь теории, является то, что эти особенности характерны не только для рациональных значений частоты возбуждения, но и для произвольных, а также возможность аналитического расчета параметров расщепления на основе уравнений Матье.

Расчеты диаграмм стабильности точным методом для рациональных значений частоты показывают, что расщепление имеет все же более сложный характер. Так, для несократимой дроби $K / P$ каждая зона стабильности разбивается на самом деле на $P-1$ полосу, даже если $K>1$. Например, при частоте $v=2 / P$ имеется слабая область нестабильности и вдоль изолинии $\beta=1 / P$. Предлагаемая здесь теория не описывает такого расщепления, так как поправки к матрице монодромии получены в первом порядке теории возмущений. При учете второго или более высоких порядков такие резонансы появятся. Однако их интенсивность очень слаба и несущественна при описании расщепления вершины первой зоны стабильности фильтра масс, так как резонансы первого порядка превалируют.

\section{2. Анализ параметров разбиения и сравнение с точными результатами}

Уравнения (26) и (29) позволяют проанализировать зависимость амплитуд возбуждения от частоты. При 


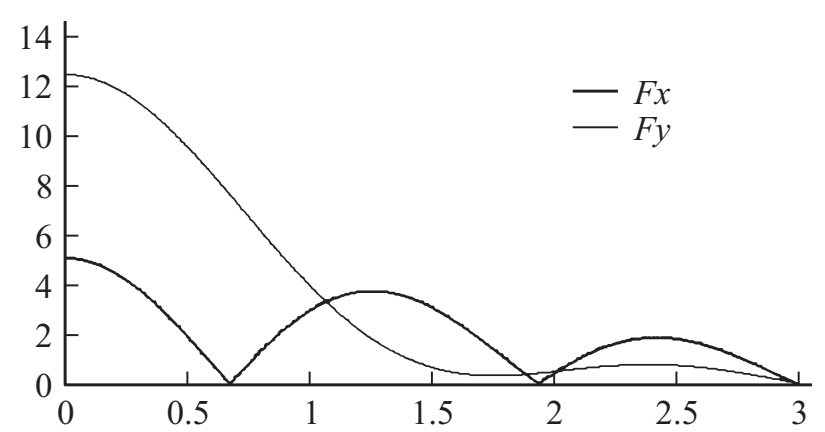

Рис. 5. Зависимость величин $F_{x}(v)$ и $F_{y}(v)$ от частоты сигнала для случая одиночного квадрупольного возбуждения.
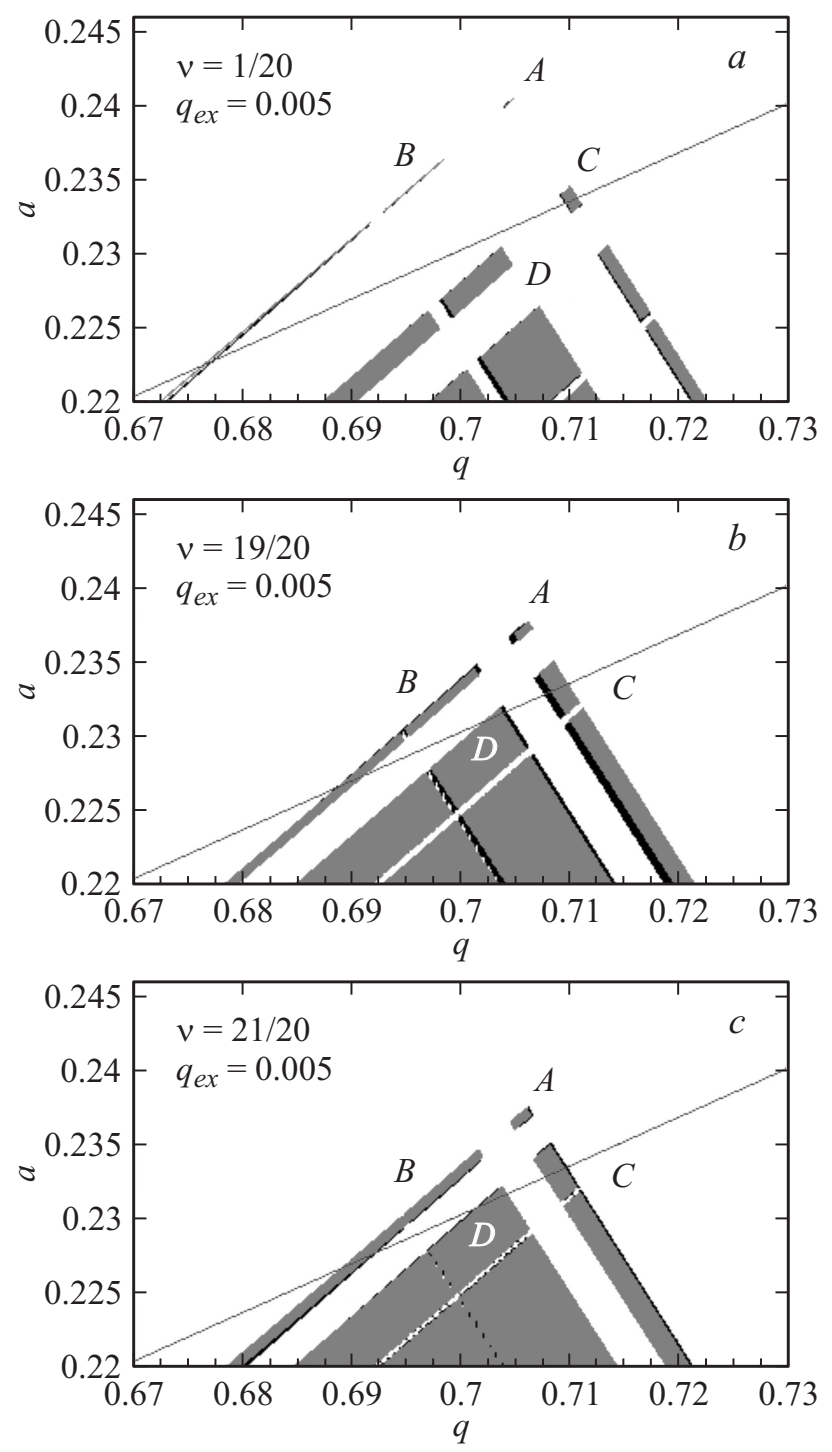

Рис. 6. Результаты расчета расщепления вершины первой зоны стабильности совместной диаграммы фильтра масс при квадрупольном возбуждении с амплитудой $q_{\mathrm{ex}}=0.005$ и различной частотой: $(a) v=1 / 20,(b) v=19 / 20,(c) v=11 / 20$. Обозначения островков стабильности $A, B, C, D$ соответствуют принятым в работе [11]. Предварительно диаграмма рассчитана теоретически и стабильные области помечены черным цветом. Вслед за этим та же диаграмма рассчитана точным методом и помечена серым цветом. Тонкой линией на каждой из диаграмм показана рабочая прямая с наклоном $\lambda=0.1645$.

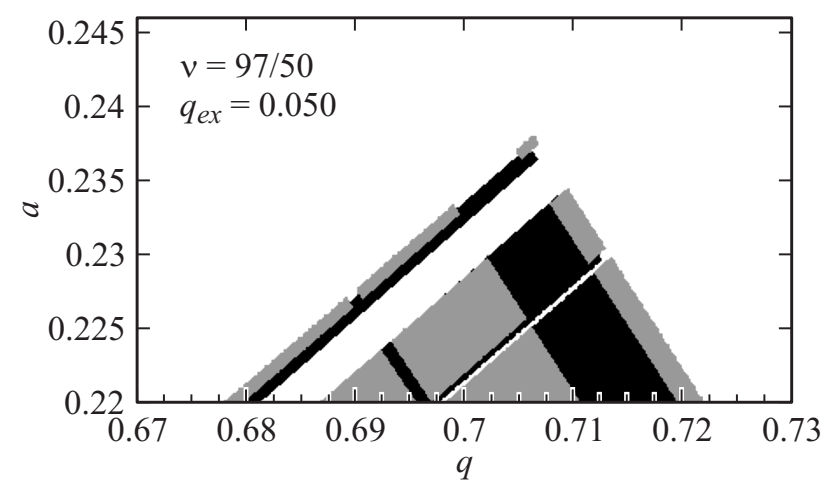

Рис. 7. Расчет расщепления вершины первой зоны при квадрупольном возбуждении с амплитудой $q_{\mathrm{ex}}=0.05$ на частоте $v=1.94$. Теоретический расчет отмечен черным цветом и покрыт точным расчетом серого цвета.

прочих одинаковых параметрах, величины $Q_{x}$ и $Q_{y}$ пропорциональны величинам $F_{x}$ и $F_{y}$, которые вычисляются из уравнения (23) в зависимости от частоты возбуждения. Расчет на основе фундаментальных решений (по поводу их вычисления см., например, работу [11]) дает зависимости, представленные на рис. 5. Из него следует, что при малых частотах возбуждения величина $F_{y}$ более чем вдвое превышает величину $F_{x}$. Это согласуется с расчетом, представленным на рис. $6, a-$ расщепление $Y$ границы зоны (слева) заметно сильнее, чем расщепление $X$ границы. При частотах немного менее единицы величина $F_{y}$ лишь немного больше $F_{x}$, а при частотах немного больше единицы они практически равны, что заметно по расщеплению границ на рис. $6, b$ и $c$. На рис. 6, $a-c$ можно видеть сравнение теоретически рассчитанной диаграммы с результатом точного расчета, из которого следует, что метод огибающей в этих случаях дает точные результаты, пригодные для количественного анализа.

Из рис. 5 следует, что обе величины $F_{x}$ и $F_{y}$ имеют исчезающе малые значения вблизи целых значений частоты 3, 4 и т.д. Это подтверждается точными расчетами, которые свидетельствуют о том, что для расщепления первой зоны возбуждениями таких высоких частот требуются довольно большие амплитуды возмушений. Очевидно тем не менее, что развиваемая здесь теория неприменима для описания таких ситуаций, так как они соответствуют резонансам высокого порядка.

Также можно видеть, что величина $F_{x}$ обращается в нуль на частотах около 0.6 и 1.94. Что касается частоты 0.6, то она никак не входит в область нашего исследования, так как вызываемые ею резонансы лежат далеко от границ стабильности. Согласно рис. 5 на частоте 1.94 расщепление границы стабильности по $X$ должно отсутствовать. Точный расчет (рис. 7) показывает, что это не так: расщепление со стороны правой границы присутствует и даже несколько значительнее, чем со стороны левой границы. Этот пример показывает, что на частотах, близких к 2 и выше, развиваемая теория 
неприменима из-за того, что резонансы в этом случае имеют более высокий порядок, чем первый. В этих случаях расщепление следует исследовать на основе матричного отображения (14) с матрицей (16) или точными методами для рациональных значений частоты. Область же применимости теории огибающей ограничить частотами, близкими к 0 или 1. Как мы увидим в дальнейшем, для наших целей этого достаточно.

\section{Выводы и заключение}

Квадрупольное возбуждение дополнительными сигналами является эффективным средством манипулирования диаграммой стабильности движения ионов в фильтре масс. Оно вызывает параметрический резонанс на секулярных частотах колебаний ионов. Эти резонансы можно описать на основе уравнений огибающей, которые сводятся к уравнениям Матье с параметрами, зависящими от амплитуды и частоты возбуждения. Под воздействием квадрупольных возбуждений совместная диаграмма разбивается на островки стабильности, положение и форму которых можно предсказать на основе уравнений огибающих с высокой точностью.

Развитая теория позволяет правильно описать характер разбиения диаграммы стабильности и количество полос нестабильности. В то же время рамки применимости теории ограничены исследованием резонансов первого порядка. Так, например, возбуждение сигналами на частотах, близких к удвоенной частоте основного ВЧ-питания (и выше) неадекватно описываются в рамках развитого формализма. В этих случаях удобно применять точные методы расчета, разработанные для возбуждений сигналами с рациональными значениями частоты.

Применения развитой теории к анализу более сложных ситуаций, например возбуждение двумя сигналами разных частот, и практические следствия для улучшения технологии фильтров масс выходят за рамки данной работы и будут изложены в следующей публикации. Метод уравнения огибающей, развитый в этой работе, создает базис для такого исследования.

Работа выполнена при поддержке Российского фонда фундаментальных исследований (грант № 14-02-97505).

\section{Список литературы}

[1] Paul W., Steinwedel $H$. // Zeitschrift für Naturforschung. 1953. Vol. A8. N 7. P. 448-450.

[2] Dawson P.H. Quadrupole Mass Spectrometry and its Applications. NY: American Institute of Physics, Woodbury, 1995. $343 \mathrm{c}$.

[3] Ding C.-F., Konenkov N.V., Douglas D.J. // Rapid Commun. Mass Spectrom. 2003. Vol. 17. C. 2495-2502.

[4] Konenkov N.V., Londry F., Ding C.-F., Douglas D.J. // J. Am. Soc. Mass Spectrom. 2006. Vol. 17. C. 1063-1073.
[5] Moradian M., Douglas D.J. // Rapid Commun. Mass Spectrom. 2007. Vol. 21. P. 3306-3310.

[6] Zhao X., Xiao Z., Douglas D.J. // Anal. Chem. 2009. Vol. 81. P. 5806-5811.

[7] Austin W.E., Holme A.E., Leck J.H. // Chapter VI. The mass filter: design and performance / Quadrupole Mass Spectrometry and Its Applications. Ed by P.H. Dawson. Amsterdam: Elsevier, 1976. P. 125-129.

[8] Dawson P.H. // Adv. Electron. Ion Optics. 1980. T. 53. P. 153-208.

[9] Titov V.V. // Int. J. Mass Spectrom. Ion Processes. 1995. Vol. 141. P. 27-35.

[10] Taylor S., Gibson J.R. // J. Mass Spectrom. 2008. Vol. 43. P. 609-616.

[11] Судаков М.Ю., Апацкая М.В. // ЖЭТФ. 2012. Т. 142. C. $1-8$.

[12] МакЛахлан Н.В. Теория и применение функций Матье. М.: ИИЛ, 1953. 474 c.

[13] Ince E. // Proceedings of the Royal Society of Edinburgh. Edinburgh: Neiland Co. Ltd., 1925-1926. Vol. XLVI. P. 20-29.

[14] Konenkov N.V, Cousins L.M., Baranov V.I., Sudakov M.Yu. // Int. J. Mass Spectrom. 2001. Vol. 208. P. 17-27.

[15] Magnus S., Winkler W. Hill's Equation. NY: John Wiley \& Sons Inc., 1966. 127 c.

[16] Мамонтов Е.В., Кирюшин Д.В. // ЖТФ. 2012. Т. 82. Вып. 9. С. 63-68.

[17] Мамонтов Е.В., Кирюшин Д.В., Журавлев В.В. // ЖТФ. 2014. Т. 84. Вып. 7. С. 110-114.

[18] Konenkov N.V., Sudakov M.Y., Douglas D.J. // J. Am. Soc. Mass Spectrom. 2002. Vol. 13. P. 597-613.

[19] Sudakov M., Apatskaya M. // Int. J. Mass Spectrom. 2012. Vol. 325-327. P. 58-66.

[20] Судаков М.Ю. // ЖТФ. 2000. Т. 70. Вып. 11. С. 108-117.

[21] Press W.H., Teukolsky S.A., Vetterling W.T., Flannery B.P. Numerical Recipes: The Art of Scientific Computing, Third Edition, NY: Cambridge University Press, 2007. 1235 p. 